\section{Jugendliche zu lebenslanger körperlicher Aktivität bewegen}

\author{
Eine kritische Betrachtung \\ bisheriger und neuerer \\ Interventionsansätze
}

\section{Originalpublikation}

Kimiecik, J., Horn, T., Newman, T. J. \& Kimiecik, C. M. (2019). Moving adolescents for a lifetime of physical activity: shifting to interventions aligned with the third health revolution. Health Psychology Review, 1-18. https: //doi.org/10.1080/ 17437199.2019.1700820

\section{Was ist zu dem Thema bereits bekannt?}

Jugendliche sind eine wichtige Zielgruppe der Bewegungs- und Sportförderung. So verfestigt sich in dieser Lebensphase die körperliche Aktivität als gesundheitsförderliche Verhaltensweise. Personen, die sich im Jugendalter körperlich betätigen, bleiben auch häufiger über die ganze Lebensspanne aktiv. Jedoch zeigen bisherige Interventionen zur Förderung der körperlichen Aktivität bei Jugendlichen nur eine moderate Wirksamkeit.

\section{Was wurde gemacht?}

Das Ziel dieses konzeptionellen Übersichtsbeitrags war daher,

1. traditionelle Interventionsansätze zur Förderung körperlicher Aktivität kritisch zu betrachten und

2. darauf aufbauend einen neueren Ansatz zur nachhaltigen Förderung von körperlicher Aktivität bei Jugendlichen zu diskutieren.

Die Mehrheit der bisherigen Interventionen verwendet einen präventionsorientierten Ansatz, der auf dem biomedizinischen Paradigma basiert ( $\triangleright$ Tab. 1 ). Dabei wird von einem mechanischen Menschenbild ausgegangen, bei dem alle Menschen in gleicher Art und Weise rational handeln.
Bei diesem Ansatz wird die körperliche Aktivität als Mittel zur Verhinderung und Behandlung von Krankheiten eingesetzt. Häufig werden universelle, nicht maßgeschneiderte Interventionen angewendet und direktiv bestimmte Bewegungsempfehlungen von Experten vorgegeben. Dementsprechend werden in diesem Interventionsansatz die individuellen Ziele, Bedürfnisse, Erfahrungen und Ressourcen sowie die subjektive Bedeutsamkeit der körperlichen Aktivität für die Jugendlichen nicht bzw. zu wenig berücksichtigt.

In einem zweiten Schritt wird ein neuer, personenbasierter Ansatz vorgestellt, der auf einem biopsychosozialen Paradigma basiert ( $\triangleright$ Tab. 1). Bei diesem Ansatz wird von einem humanistischen Menschenbild ausgegangen, bei dem das Individuum und seine Entwicklung aus einer ganzheitlichen Perspektive betrachtet wird.
Dabei werden die Ressourcen und das persönliche Wachstum eines Individuums sehr viel stärker berücksichtigt. So streben Jugendliche z. B. nach positiven Erfahrungen und (Wohl-)befinden bei körperlichen Aktivitäten. Diese positiven inneren Prozesse wiederum tragen dazu bei, dass Jugendliche langfristig motiviert sind, körperlich aktiv zu bleiben.

\section{ZUSATZINFO}

Der Begriff personenbasierter Ansatz wird häufig synonym zum personenzentrierten und personenorientierten Ansatz verwendet. Die Ansätze sind sich inhaltlich sehr ähnlich (z. B. vergleichbares Menschenbild), jedoch nicht identisch. Für eine ausführlichere Diskussion vgl. z. B. Laursen (2015) und Valsnier (2015).

>Tab. 1 Vergleich verschiedener Interventionsansätze zur Förderung der körperlichen Aktivität bei Jugendlichen.

\begin{tabular}{|c|c|c|}
\hline & Präventionsorientierter Ansatz & Personenbasierter Ansatz \\
\hline Ursprünge & $\begin{array}{l}\text { - Mechanisches Menschenbild } \\
\text { - Biomedizinisches Paradigma }\end{array}$ & $\begin{array}{l}\text { - Humanistisches Menschenbild } \\
\text { - Positive Psychologie, Youth Deve- } \\
\text { lopment und } \\
\text { Entwicklungswissenschaft } \\
\text { - Biopsychosoziales Paradigma }\end{array}$ \\
\hline $\begin{array}{l}\text { Ausgangs- } \\
\text { punkt }\end{array}$ & $\begin{array}{l}\text { - Defizitorientiert } \\
\text { - Verhaltensfokussiert }\end{array}$ & $\begin{array}{l}\text { - Wachstums- und } \\
\text { ressourcenorientiert } \\
\text { - Individuumsfokussiert }\end{array}$ \\
\hline $\begin{array}{l}\text { Primäre } \\
\text { Annahme }\end{array}$ & $\begin{array}{l}\text { - Jugendliche handeln rational } \\
\text { und Interventionen werden } \\
\text { benötigt, um Defizite } \\
\text { auszugleichen }\end{array}$ & $\begin{array}{l}\text { - Jugendliche streben nach Wohlbe- } \\
\text { finden und gesunden } \\
\text { Verhaltensweisen }\end{array}$ \\
\hline Schwerpunkt & $\begin{array}{l}\text { Körperliche Aktivität als Mittel } \\
\text { zur Verringerung des Krank- } \\
\text { heitsrisikos und Behandlung } \\
\text { von Krankheiten }\end{array}$ & $\begin{array}{l}\text { - Das Erfahren von positiven Aspek- } \\
\text { ten des menschlichen Lebens (z. B. } \\
\text { Selbstbestimmung) führt zu nach- } \\
\text { haltigerem Gesundheitsverhalten } \\
\text { (z. B. körperlicher Aktivität) }\end{array}$ \\
\hline $\begin{array}{l}\text { Verhaltensver- } \\
\text { änderungspro- } \\
\text { zess }\end{array}$ & $\begin{array}{l}\text { - Universelle, nicht maßge- } \\
\text { schneiderte Interventionen } \\
\text { mit von ExpertInnen vorge- } \\
\text { schriebenen } \\
\text { Bewegungsempfehlungen }\end{array}$ & $\begin{array}{l}\text { - Autonomieförderliche Umgebun- } \\
\text { gen zur Selbstexploration und -ent- } \\
\text { faltung schaffen, damit Jugendliche } \\
\text { ihre individuellen Vorlieben und } \\
\text { Stärken entdecken können }\end{array}$ \\
\hline Limitationen & $\begin{array}{l}\text { - Zu einfach } \\
\text { - Verhalten ohne Bedeutung }\end{array}$ & $\begin{array}{l}\text { - Zu komplex } \\
\text { - Bedeutung von körperlicher Aktivi- } \\
\text { tät keine hinreichende Bedingung } \\
\text { für Verhaltensänderung }\end{array}$ \\
\hline
\end{tabular}

Notiz. Angepasste Tabelle von Kimiecik, Horn, Newman und Kimiecik (2019, S. 5). 


\section{Was bedeuten die Ergebnisse für die Praxis?}

Für die Praxis verdeutlicht dieser Beitrag, dass ein autonomieförderliches Umfeld (z. B. Eltern, TrainerInnen) geschaffen werden sollte, das die Selbstexploration und -entfaltung der Jugendlichen fördert und innen dadurch positive Erfahrungen und Emotionen ermöglicht. Beispielsweise sollten die individuellen Bedürfnisse, Motive und Ziele stärker berücksichtigt werden, in dem die Jugendlichen körperliche Aktivitäten ausprobieren und herauszufinden können, welche Aktivitäten ihnen besonders Freude bereiten.

\section{Was bedeuten die Ergebnisse für die Forschung?}

Die Kombination des personenbasierten und des präventionsorientierten Ansatzes kann die Wirksamkeit von Interventionen zur Förderung der körperlichen Aktivität bei Jugendlichen erhöhen. Interventionen sollten daher nicht nur direkt auf die Erhöhung der körperlichen Aktivität ausgerichtet sein, sondern auch auf die Vermittlung von positiven Erfahrungen und Emotionen während einer Aktivität, damit Jugendliche motiviert sind eine Aktivität zu beginnen und diese aufrechtzuerhalten. Beispielsweise sollte der Fokus bei übergewichtigen Jugendlichen nicht nur auf der Reduktion des Körpergewichts durch Bewegung liegen (präventionsorientierter Ansatz), sondern auch darauf, dass die Jugendlichen während einer körperlichen Aktivität ein gutes Befinden haben (personenbasierter Ansatz).

\section{Fazit}

Der beschriebene konzeptionelle Übersichtsbeitrag bietet eine Erklärung, warum gewisse Interventionen zur Förderung der körperlichen Aktivität bei Jugendlichen nur beschränkt wirksam sind. Zudem regt der Beitrag an, einen Paradigmawechsel bei der Konzeption von Interventionen vorzunehmen. Zukünftige Interventionen für Jugendliche sollten nicht ausschließlich auf die Krankheit fokussieren, sondern vielmehr das Individuum mit seinen Ressourcen und seiner Entwicklung betrachten. Insbesondere sollten vermehrt positive Erfahrungen vermittelt sowie das Wohlbefindens von Jugendlichen gefördert werden.

\section{Korrespondenzadresse}

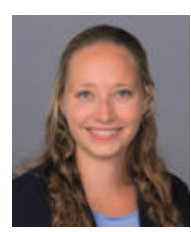

Vanessa Gut

Institut für Sportwissenschaft, Universität Bern Bremgartenstraße 145 3012 Bern

Schweiz vanessa.gut@ispw.unibe.ch 